\title{
Process-Independent Resistor Temperature-Coefficients using Series/Parallel and Parallel/Series Composite Resistors
}

\author{
B. Robert Gregoire and Un-Ku Moon \\ Electrical Engineering and Computer Science \\ Oregon State University, Corvallis, OR
}

\begin{abstract}
- this paper introduces series/parallel and parallel/series composite resistor topologies. These topologies allow one to design a temperature coefficient that is insensitive to process variations, unlike the traditional simple series and simple parallel composite resistors. Formulas and normalized circuits are provided. Four zero-temperature coefficient resistors are simulated, and methods for picking the best topology are discussed.
\end{abstract}

\section{INTRODUCTION}

It is often desirable to create a resistor with a particular temperature coefficient. This can be done with a series composite resistor (Fig. 1a) or a parallel composite resistor (Fig. 1b). Such configurations are well known [1]-[5], with the series composite resistor being the most common. Unfortunately, the temperature coefficients of these configurations depend on sheet resistance processes corners. Two process independent configurations (series/parallel and parallel/series) are the subject of this paper.

Section II reviews resistor temperature coefficients, and explains the terms used in the paper. Section III reviews the simple series and simple parallel resistors, and highlights their problems when sheet resistances vary independently. Section IV introduces the series/parallel and parallel/series composite resistors. Simulations and a discussion on the best topology is covered in section $\mathrm{V}$.

\section{RESISTOR DEFINITIONS}

A resistor's temperature behavior can be expressed in terms of its temperature coefficients $\mathrm{TC}_{1}$ and $\mathrm{TC}_{2}$, which are derived from the Taylor Series expansion around the nominal temperature $\mathrm{T}_{0}$ :

$$
\mathrm{R}(\mathrm{T})=\mathrm{R}\left(\mathrm{T}_{0}\right) *\left(1+\mathrm{TC}_{1} *\left(\mathrm{~T}-\mathrm{T}_{0}\right)+\mathrm{TC}_{2} *\left(\mathrm{~T}-\mathrm{T}_{0}\right)^{2}\right)
$$

$\mathrm{R}\left(\mathrm{T}_{0}\right)$ will change with process. This paper assumes $\mathrm{TC}_{1}$ and $\mathrm{TC}_{2}$ do not change with process, but the ideas presented

This work is supported in part by the Semiconductor Research Corporation under contract 2005-HJ-1308 can be adapted to accommodate $\mathrm{TC}_{1}$ variations. $\mathrm{TC}_{2}$ variations do not affect the results of this paper.<smiles>[R8]C(C)C#CC(C)C#C</smiles>

(a)

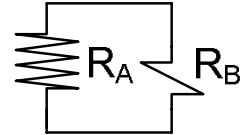

(b)
Fig. 1. Composite resistors: (a) Simple series and (b) simple parallel. $R_{A}$ and $R_{B}$ are resistors with different temperature coefficients, and are weighted to give a particular temperature coefficient to the combination.

The following definitions and resistors will be used in this paper.

\section{TABle 1. Definitions}

\begin{tabular}{|l|l|}
\hline Name & Definition \\
\hline $\mathrm{R}_{\mathrm{A}}$ & Resistor with temperature coefficient $\mathrm{TC}_{\mathrm{A}}$ \\
$\mathrm{R}_{\mathrm{B}}$ & Resistor with temperature coefficient $\mathrm{TC}_{\mathrm{B}}$ \\
$\mathrm{TC}_{\mathrm{A}}$ & $\frac{1}{\mathrm{R}_{\mathrm{A}}} \frac{\partial \mathrm{R}_{\mathrm{A}}}{\partial \mathrm{T}} \quad\left(1^{\text {st }}\right.$ Order Temp-coefficient of $\left.\mathrm{R}_{\mathrm{A}}\right)$ \\
$\mathrm{TC}_{\mathrm{B}}$ & $\frac{1}{\mathrm{R}_{\mathrm{B}}} \frac{\partial \mathrm{R}_{\mathrm{B}}}{\partial \mathrm{T}} \quad\left(1^{\text {st }}\right.$ Order Temp-Coefficient of $\left.\mathrm{R}_{\mathrm{B}}\right)$ \\
$\beta$ & $\mathrm{R}_{\mathrm{A}} / \mathrm{R}_{\mathrm{B}}$ at $\mathrm{T}_{0}$ \\
$\mathrm{~T}_{0}$ & Nominal Temperature \\
$\alpha$ & Scalar constant \\
\hline
\end{tabular}

TABLE 2. RESISTOR PARAMETERS USED IN THIS PAPER.

\begin{tabular}{|c|cc|}
\hline Name & $\left.\mathbf{T C}_{\mathbf{1}} \mathbf{( p p m} / \mathbf{C}\right)$ & $\left.\mathbf{T C}_{\mathbf{2}} \mathbf{( p p m} / \mathbf{C}^{\mathbf{2}}\right)$ \\
\hline $\mathrm{R}_{\text {UTC }}$ & 1200 & 0.4 \\
$\mathrm{R}_{\text {LTC }}$ & -700 & 0.9 \\
\hline
\end{tabular}


The subscripts UTC and LTC stand for "Upper Temperature Coefficient" and "Lower Temperature Coefficient" respectively. The composite resistor temperature coefficient must be between UTC and LTC.

The resistors are representative of low-sheet and highsheet poly resistors that one would find in a modern CMOS process. The second order coefficients are included although they do not affect the calculations.

\section{Simple COMPOSITE Resistors}

\section{A. Temperature coefficient}

It is straight forward to show that the temperature coefficient of the simple series composite resistor (Fig. 1a) is

$$
\mathrm{TC}_{\mathrm{s}}=\mathrm{TC}_{\mathrm{A}} \frac{\beta}{1+\beta}+\mathrm{TC}_{\mathrm{B}} \frac{1}{1+\beta} .
$$

Similarly, the temperature coefficient of the simple parallel composite resistor (Fig. 1b) is

$$
\mathrm{TC}_{\mathrm{p}}=\mathrm{TC}_{\mathrm{A}} \frac{1}{1+\beta}+\mathrm{TC}_{\mathrm{B}} \frac{\beta}{1+\beta} .
$$

The composite resistor temperature coefficient is determined by the relative size of $R_{A}$ and $R_{B}$ (remember $\beta=R_{A} / R_{B}$ at $T_{0}$ ). Any temperature coefficient between $T_{C A}$ and $\mathrm{T}_{\mathrm{CB}}$ can be obtained.

\section{B. Simple composite resistors over process corners}

The temperature coefficients (2) and (3) remain unchanged if the sheet resistance of $R_{A}$ and $R_{B}$ change equal amounts. However, the sheet resistances of $R_{A}$ and $R_{B}$ are usually uncorrelated, meaning that the sheet resistance of $\mathrm{R}_{\mathrm{A}}$ can be high, while the sheet resistance of $R_{B}$ is low. This will change the value of the temperature coefficient since $\beta$ will change. One could intuit this behavior by noting that, as the value of $R_{B}$ increases relative to $R_{A}$, the temperature coefficient of the simple series composite resistor will be closer to the temperature coefficient of $R_{B}$. On the other hand, the simple parallel composite resistor will behave more like $\mathrm{R}_{\mathrm{A}}$ as $\mathrm{R}_{\mathrm{B}}$ increases.

To illustrate the process dependence of the series/parallel and parallel/series resistors, the upper temperature coefficient (UTC) and lower temperature coefficient (LTC) resistors in Table 2 were used to construct the $1 \mathrm{k} \Omega$ resistors with zero temperature coefficients. The values are shown in Fig. 2.

Zero temperature coefficient was chosen for simplicity; composite resistors can be used to produce any temperature coefficient between the first order coefficients of the two resistor types (1200 and $-700 \mathrm{ppm} / \mathrm{C}$ in this case).

Fig. 3 shows that the overall temperature coefficient is a strong function of skewed process corners. With the $\mathrm{R}_{\mathrm{UTC}}$ sheet resistance $20 \%$ high, and the $\mathrm{R}_{\mathrm{LTC}}$ sheet resistance $20 \%$ low, the overall temperature coefficient is $+200 \mathrm{ppm} / \mathrm{C}$

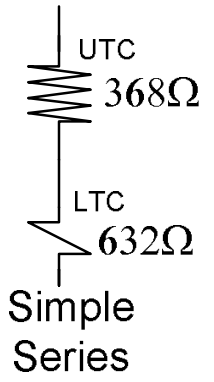

(a)

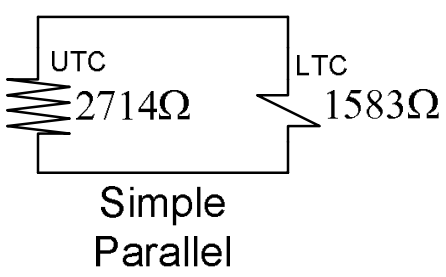

(b)
Fig. 2. Simple series (a) and simple parallel (b) $1 \mathrm{k} \Omega$ composite resistors designed for zero temperature coefficients.

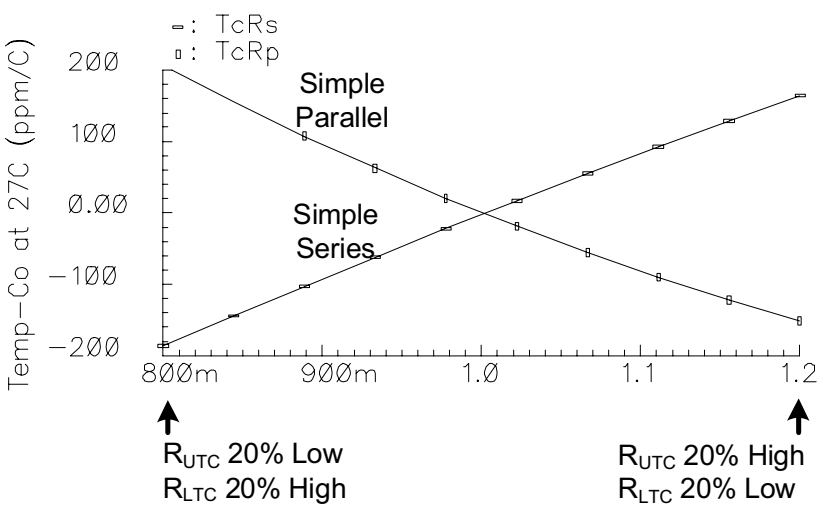

Fig. 3. Temperature Coefficient of simple series and simple parallel composite resistors showing dependence on the independent process corners of $R_{A}$ and $R_{B}$.

for the simple series composite, and $-200 \mathrm{ppm} / \mathrm{C}$ for the simple parallel composite.

Since the circuits in Fig. 2 react oppositely to changes in the relative sizes of $R_{U T C}$ and $R_{L T C}$, one could expect a combination resistor using both series and parallel components could be made that is independent of the relative sizes of $R_{U T C}$ and $R_{L T C}$. That is the subject of the next section.

\section{Series/Parallel and Parallel/Series COMPOSITE RESISTORS}

\section{A. Series-with-parallel-compensation (series/parallel)}

The series-with-parallel-compensation resistor is shown in Fig. 4a. The name reflects the simple series composite resistor formed by $R_{A}$ and $R_{B}$ with a parallel resistor added to compensate for sheet resistance process variations in $R_{A}$ and $R_{B}$. It will be referred to as series/parallel. The insensitivity to sheet resistance variations can be seen by noting that, as $R_{B}$ increases relative to $R_{A}$, its contribution to the temperature coefficient in the left leg increases. At the same time, the right leg contribution to the overall temperature coefficient decreases. Thus the two legs have opposite sensitivities to process variations in sheet resistance. The temperature coefficient of the series/parallel 


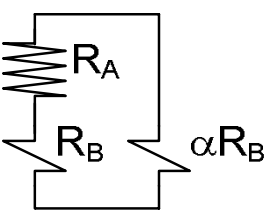

(a)

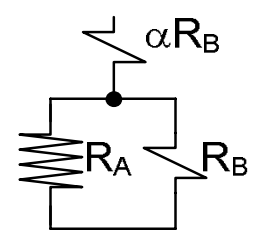

(b)
Fig. 4. Two composite resistor configurations that can be tuned to have a temperature coefficient that is independent of process corners. (a) series with parallel compensation (series/parallel) and (b) parallel with series compensation (parallel/series). In both circuits, $\alpha R_{B}$ is the compensation resistor.

resistor is

$$
T C_{s p}=T C_{B}+\frac{\left(T C_{A}-T C_{B}\right)\left(\alpha_{s p} \beta_{s p}\right)}{\left(1+\beta_{s p}\right)\left(1+\alpha_{s p}+\beta_{s p}\right)} .
$$

The "sp" subscript denotes series/parallel. The value of $\beta_{\text {sp }}$ (ratio of $R_{A}$ to $R_{B}$ ) that makes (4) independent of process variations (i.e. changes in $\beta_{\mathrm{sp}}$ ) can be found by taking the derivative of (4) with respect to $\beta_{\mathrm{sp}}$ and setting it to zero.

Doing so results in

$$
\beta_{\mathrm{sp}}^{2}=\alpha_{\mathrm{sp}}+1
$$

Substituting (5) into (4) gives

$$
\beta_{\mathrm{sp}}=\frac{\mathrm{TC}_{\mathrm{A}}+\mathrm{TC}_{\mathrm{sp}}-2 \mathrm{TC}_{\mathrm{B}}}{\mathrm{TC}_{\mathrm{A}}-\mathrm{TC}_{\mathrm{sp}}} .
$$

Thus series/parallel ratios $\alpha_{\mathrm{sp}}$ and $\beta_{\mathrm{sp}}$ can be determined from (5) and (6).

\section{B. Parallel-with-series-compensation (parallel/series)}

The parallel-with-series-compensation is shown in Fig. 4b. The name reflects the simple parallel composite resistor formed by $R_{A}$ and $R_{B}$ which is compensated by a series resistor $\alpha R_{B}$. As the sheet resistance of $R_{B}$ increases relative to $R_{A}$, the parallel contribution of $R_{B}$ will contribute less to the overall temperature coefficient. This will be compensated by the series contribution $\alpha \mathrm{R}_{\mathrm{B}}$ will contribute more. The temperature coefficient for the parallel/series resistor is

$$
\mathrm{TC}_{\mathrm{ps}}=\mathrm{TC}_{\mathrm{B}}+\frac{\left(\mathrm{TC}_{\mathrm{A}}-\mathrm{TC}_{\mathrm{B}}\right) /\left(\beta_{\mathrm{ps}} \alpha_{\mathrm{ps}}\right)}{\left(1+\frac{1}{\beta_{\mathrm{ps}}}\right)\left(1+\frac{1}{\alpha_{\mathrm{ps}}}+\frac{1}{\beta_{\mathrm{ps}}}\right)} .
$$

Taking the derivative of (7) with respect to $\beta_{\mathrm{ps}}$ and setting it to zero will result in the value of $\beta_{\mathrm{ps}}$ that will make (7) independent of $R_{A}$ and $R_{B}$ sheet resistance process variations. This value is

$$
\beta_{\mathrm{ps}}^{2}=\frac{\alpha_{\mathrm{ps}}}{\alpha_{\mathrm{ps}}+1},
$$

Substituting (8) into (7) gives

$$
\beta_{\mathrm{ps}}=\frac{\mathrm{TC}_{\mathrm{A}}-\mathrm{TC}_{\mathrm{ps}}}{\mathrm{TC}_{\mathrm{A}}+\mathrm{TC}_{\mathrm{ps}}-2 \mathrm{TC}_{\mathrm{B}}} .
$$

Thus, the parallel/series ratios $\beta_{\mathrm{ps}}$ and $\alpha_{\mathrm{ps}}$ can be determined from (8) and (9).

\section{Generalized form}

Equations (4)-(9) can be used to construct the normalized circuits in Fig. 5. So, once the desired temperature coefficient is known, one can simply plug in the numbers into Fig. 5 to determine normalized circuit values.

\section{Simulation of a Zero Temperature COEFFICIENT1K- $\Omega$ RESISTOR}

\section{A. The four possible configurations}

There are four possible ways to create a $1-\mathrm{k} \Omega$ zero temperature-coefficient resistor with series/parallel and parallel/series methods. (The compensating resistor can be either UTC or LTC for both series/parallel and parallel/series). They are shown in Fig. 6, with a different symbol for the UTC and LTC resistors. Note that the number of significant digits is only to allow the reader to verify calculations. After all, the purpose of this paper is to give a topology that is insensitive to exact resistor values.

\section{B. Simulation Results}

The four resistors in Fig. 6 were simulated over temperature while skewing the relative values of the UTC and LTC resistors. This changes the value of $\beta$. ( $\alpha$ is assumed constant since it is the ratio of two resistors of the same type, and can be well controlled.) The temperature coefficient was then calculated at $\mathrm{T}_{0}(27 \mathrm{C})$. This simulated how the temperature coefficient would change over
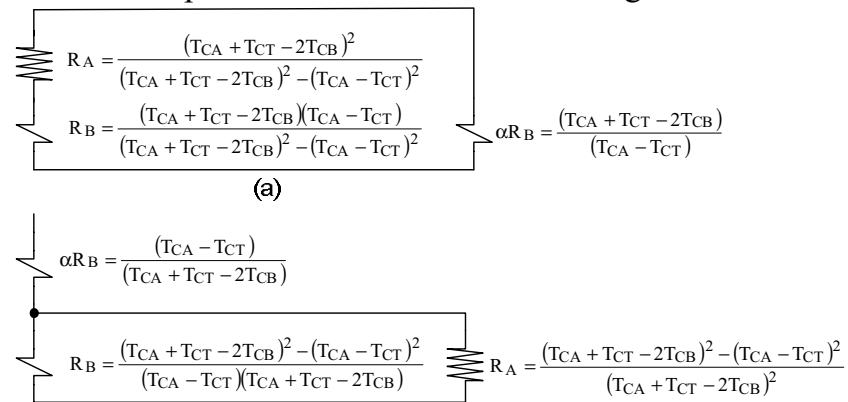

(b)

Fig. 5. (a) series/parallel and (b) parallel/series resistors normalized to $1 \mathrm{ohm}$, and total temperature coefficient of $\mathbf{T}_{\mathbf{C T}}$.
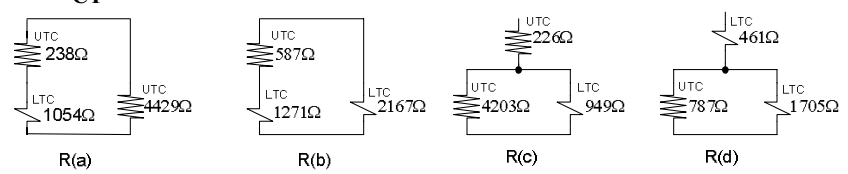

Fig. 6. Resistors for simulation of the four possible ways of constructing zero temperature coefficient $1 \mathrm{k}-\Omega$ series/parallel and parallel/series resistors from two resistor types (UTC and LTC). The temperature coefficients of UTC and LTC are given in Table 2. 
independent process variations of UTC and LTC sheet resistance.

As can be seen from Fig. 7 the variation in temperature coefficient for the series/parallel resistor is a factor of six less than the simple composite resistors (Fig. 3). Also notable is that the variation $\mathrm{R}(\mathrm{a})+\mathrm{R}(\mathrm{b})$ is a factor of fifty less than the simple composites.

Fig. 8 shows the performance of the parallel/series resistor. The behavior when the resistances are skewed is very similar to the series/parallel circuit. This is not surprising given the inverse symmetry of (4) and (7).

For the both the parallel/series and the series/parallel resistors the temperature coefficient moves toward the coefficient of the compensation resistor as the UTC and LTC resistors are skewed from their optimal weightings.

\section{The best resistor topology}

The composite resistors $\mathrm{R}(\mathrm{a})$ through $\mathrm{R}(\mathrm{d})$ have similar performance when their component resistance values are skewed. The performance can be further improved by choosing one of the possible sums (not necessarily equally weighted): $R(a)+R(b), R(a)+R(d), R(b)+R(c)$ or $R(d)+R(c)$, which, again, perform similarly. Thus, the main consideration in choosing a topology is not the temperature coefficient performance. Instead, other factors, such as total area, determine the best choice. Area used will depend on the particular process, but a feel for the spread in the Fig. 6 designs can be obtained by noting the total resistance of each topology.

Table 3 shows the total resistance of each topology. Obviously the simple series is the most efficient, but it has poor performance when the resistances are skewed. For this example, the best resistor is probably $\mathrm{R}(\mathrm{d})$ since it requires the least resistance of the compensated forms. This could change depending on minimum widths and relative sheet resistances of the process.

Sums of resistances (e.g. $\mathrm{R}(\mathrm{d})+\mathrm{R}(\mathrm{c})$ ) offer tightest control of the temperature coefficient.

Table 3. Summary of total resistances required for the series/parallel and parallel/series composites.

\begin{tabular}{|l|c|}
\hline Name & $\begin{array}{l}\text { Total resistance used to } \\
\text { create the } 1 \mathrm{k} \Omega \text { resistor }\end{array}$ \\
\hline $\mathrm{R}(\mathrm{a})$ & 5720 \\
$\mathrm{R}(\mathrm{b})$ & 4023 \\
$\mathrm{R}(\mathrm{c})$ & 5377 \\
$\mathrm{R}(\mathrm{d})$ & 2953 \\
Simple series & 1000 \\
Simple parallel & 4297 \\
\hline
\end{tabular}

VI. CONCLUSION

The temperature coefficient of simple series and simple

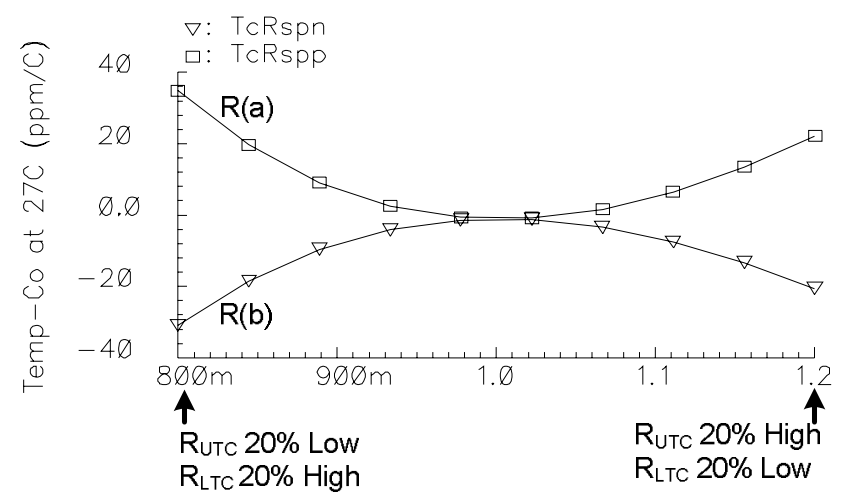

Fig. 7. Series/parallel temperature coefficient as a function of UTC/LTC sheet resistance skew.

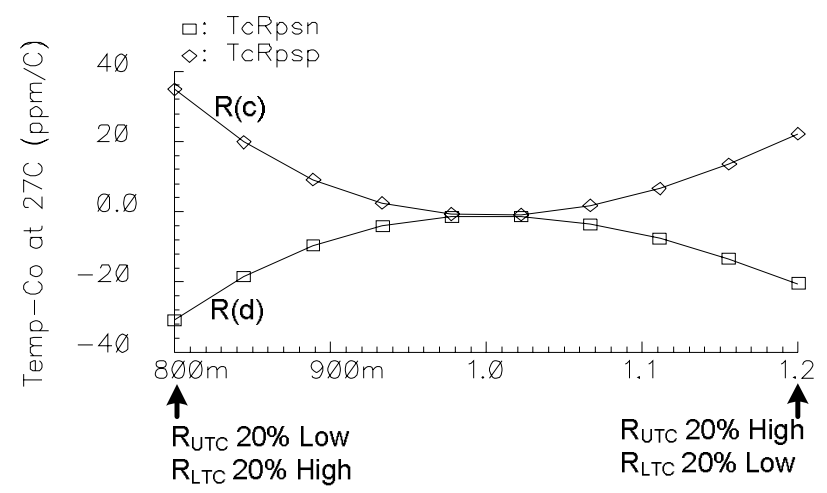

Fig. 8. Parallel/series temperature coefficient as a function of UTC/LTC sheet resistance skew.

parallel are sensitive to independent variations of the sheetresistance. Series/parallel and parallel/series topologies can be used to construct a resistance with minimal temperature coefficient change over process corners. The resistance can be realized four different ways. None of the four topologies are inherently more robust, and other factors, such as area, will dictate the best topology. Linear combinations of the resistors can give further improvement in temperature coefficient stability over process corners.

\section{REFERENCES}

[1] A.P. Brokaw, "Band-gap voltage reference with curvature correction," U.S. Patent 4,250,445, Feb. 10, 1981.

[2] M. de Wit, "Temperature independent resistor," U.S. Patent 5,448,103, Sep. 5, 1995.

[3] J.M. Laraia, "Circuits and methods for providing a current refrence with a controlled temperature coefficient using a series composite resistor," U.S. Patent 6,351,111, Feb. 26, 2002.

[4] J.M. Laraia, "Circuits and methods for providing a bandgap voltage reference using composite resistors," U.S. Patent 6,342,781, Jan. 29, 2002

[5] J. Lu, Y. Wang, N. Xu, and M. Gao, "Temperature Compensation in Bootstrapped Current Reference Source," 2003 IEEE Conf. Elec. Dev. Solid-State Circ., Dec. 2003, pp. 491-494. 